\title{
Untersuchungen zur Mortalität von Legehennen gehalten in Einzel- und Gruppenkäfigen
}

\author{
Herrn Professor Dr. Dr. h.c. mult. Ernst Kalm zum 65. Geburtstag gewidmet
}

\begin{abstract}
Title of the paper: Mortality of laying hens housed in single and group cages

This study provides genetic parameters for mortality of laying hens estimated with linear and threshold models. Records of one line from a commercial breeding programme of White Leghorns from three generations were available. Data included observations of 8636 hens from single and from 6908 hens of group cages. Mortality was defined as death in the first six months of lay with dead $=1$ and alive $=0$. The average mortality was $3.3 \%$ in single and $6.3 \%$ in group cages. The binary traits were analysed separately by linear animal (LAM), by threshold animal (TAM) and by threshold sire models. Further the two mortalities were analysed together by a linear-linear animal (LLAM) and threshold-threshold animal model (TTAM). The LLAM estimates of heritability were 1.5\% for single and 3.2\% for group cages. The heritability estimates of TTAM ranged from $9.6 \%$ to $9.9 \%$. The rank correlations between breeding values of LAM and TAM were for all sires, the $10 \%$ best and the $5 \%$ best sires in the range 0.96 to 1.00 . The analysis of rank correlations of the linear and threshold models showed: The LLAM provides a good (but only suboptimal) alternative for breeding value estimation of mortality in the investigated laying hen population.
\end{abstract}

Key Words: laying hens, mortality, heritability, breeding values, single and group cages

\section{Zusammenfassung}

In dieser Studie wurden genetische Parameter für die Mortalität von Legehennen mit Hilfe von linearen und Schwellenwertmodellen geschätzt. Für die Untersuchungen standen Daten einer kommerziellen Weißen Leghornlinie aus 3 Generationen zur Verfügung. Mortalitätsdaten von 8636 Hennen aus Einzelkäfig- und von 6908 Hennen aus Gruppenkäfighaltung wurden ausgewertet. Die Mortalität wurde innerhalb der ersten sechs Legemonate als binäres Merkmal definiert mit den Ausprägungen Eins für gestorbene und Null für überlebende Henne. Die mittleren Mortalitäten im Einzel- bzw. Gruppenkäfig zeigten mit 3,3\% bzw. 6,3\% relativ niedrige Werte. Die Schätzung der Heritabilitäten und Zuchtwerte für die binären Merkmale erfolgte einerseits separat mit linearen Tiermodellen (LAM), mit Schwellenwerttier-(TAM) und mit Schwellenwertvatermodellen (TSM). Andererseits wurden beide Abgangsraten gemeinsam mit Zweimerkmals-Tiermodellen für lineare (LLAM) und binäre (TTAM) Merkmale analysiert. Die Schätzungen für die Heritabilität durch LLAM lagen für Einzel- bzw. Gruppenkäfige bei 1,5 bzw. 3,2\%. Das TTAM lieferte für die Mortalitäten $\mathrm{h}^{2}$-Werte von 9,6\% und 9,9\%. Zwischen den Zuchtwerten der Modelle LAM und TAM wurden für alle Väter, die 10\%-besten und 5\%-besten Väter hohe Werte zwischen 0,96 und 1,00 geschätzt. Die Analyse der Rangkorrelationen zwischen den linearen und den Threshold-Modellen zeigte, dass das bivariate lineare Modell einen brauchbaren (aber nur suboptimalen) Kompromiss zur Zuchtwertschätzung für die Mortalität in der untersuchten Legehennenlinie darstellt.

Schlüsselwörter: Legehennen, Mortalität, Heritabilität, Zuchtwerte, Einzel- und Gruppenkäfige

1.

\section{Einleitung}

In jüngerer Zeit haben die Autoren und der zu ehrende Jubilar mehrere Arbeiten zur Schätzung genetischer Parameter von Legeleistungen unter dem Aspekt der Einzelbzw. Gruppenhaltung vorgestellt (MIELENZ u.a., 2003; NURGIARTININGSIH u.a., 
2002; SAVAS u.a., 1998). Vorliegender Beitrag beschäftigt sich mit der Mortalität von Legehennen. In Zuchtprogrammen für das Legehuhn ist man bestrebt diese auf ein Minimum zu reduzieren. Eine geringe Mortalitätsrate ist Voraussetzung für eine hohe Anzahl absatzfähiger Eier pro eingestallter Henne. Die mittlere Mortalität ist folglich in kommerziellen Legehennenlinien relativ gering. Sie liegt unter kontrollierten Bedingungen, wie sie durch die Käfighaltung gegeben ist, bei rund 0,1\% pro Woche und rund 5\% pro Jahr (PREISINGER, 1998). Durch die direkte oder indirekte Selektion auf hohe Legeleistung pro eingestallter Henne bzw. aufgrund der Tatsache, dass nur von überlebenden Hennen Nachkommen erzeugt werden können, befindet sich die Mortalität unter permanentem Selektionsdruck. Eine direkte Selektion ist nur wenig effektiv, da die Mortalität unter kontrollierten, wenig stressbehafteten Umweltbedingungen eine sehr geringe Heritabilität besitzt. In der grundlegenden Arbeit von ROBERTSON und LERNER (1949) wurden für die Mortalität in Abhängigkeit von der Todesursache $\mathrm{h}^{2}$-Werte zwischen 2,6 und 8,9\% gefunden. Bedingt durch die geringen mittleren Mortalitäten in Zuchtpopulationen können sich Unterschiede innerhalb und zwischen Familien nur unzureichend ausprägen. Durch die Haltung der Hennen in Gruppenkäfigen mit 4 Tieren pro Käfig ist ein Anstieg der Mortalität im Vergleich zur Haltung in Einzelkäfigen zu erwarten. Eine Ursache für diese Zunahme ist die Konkurrenzsituation der Tiere innerhalb eines Käfigs zum Beispiel um Futter und Wasser.

Die Beschreibung der Mortalität von Individuen als (0,1)-Merkmal zu einem festen Zeitpunkt besitzt den Nachteil, dass die Information über den Abgangszeitpunkt verloren geht. Dagegen beschreibt die Lebensdaueranalyse die Veränderung der Abgangsrate über die gesamte Produktionsperiode. DUCROCQ u.a. (2000) führten eine Lebensdaueranalyse für die Aufzucht- und Produktionsphase einer großen Legehennenlinie separat durch. Für die Auswertung wurde ein Cox-Hazard-Modell mit zufälligen Vater- und Muttereffekten angepasst. Die Einbettung von Lebensdaueranalysen in die routinemäßige Zuchtwertschätzung ist eine große (noch ungelöste) rechentechnische Herausforderung. Ziel dieser Arbeit ist es Heritabilitäten für die Mortalität im Einzelund Gruppenkäfig zu schätzen. Dabei wird der klassische lineare Ansatz als auch das Schwellenwertkonzept verfolgt. Neben der Schätzung von genetischen Parametern wird die Zuchtwertschätzung mit dem approximativen linearen Ansatz mit der Behandlung der Mortalität als latente, nichtbeobachtbare Variable im Schwellenwertmodell verglichen. Die hier zur Auswertung der Mortalität verwendeten Ein- und Zweimerkmalsmodelle lassen sich teilweise einfach zu einer simultanen Zuchtwertschätzung für Leistungs- und Mortalitätsmerkmale erweitern.

2. Material

Zur Untersuchung der Mortalität im Einzel- und Gruppenkäfig standen Daten einer Weißen Leghorn Linie aus 3 Generationen zur Verfügung. Pro Generation wurden 4 Schlüpfe erzeugt und die Hennen aus jeweils 2 Schlüpfen in Einzelkäfige (mit $900 \mathrm{~cm}^{2}$ pro Henne) und 4-er Gruppenkäfige (mit $450 \mathrm{~cm}^{2}$ pro Henne) eingestallt. Insgesamt existieren 229 Väter mit durchschnittlich 36 Nachkommen in Einzel- und 28 Töchtern in Gruppenkäfigen. Das hohe Leistungsniveau für die Eiproduktionsmerkmale kann in der Arbeit von NURGIARTININGSIH u.a. (2004) nachgelesen werden.

Die Mortalität wurde als binäres Merkmal definiert mit dem Wert Eins falls eine Henne innerhalb der ersten sechs Legemonate gestorben ist und dem Wert Null für 
überlebende Henne. Die Untersuchungen zur Abgangsrate erfolgten sowohl für Hennen gehalten im Einzel- als auch für Hennen gehalten im Gruppenkäfig, wobei nur Käfige mit 4 eingestallten Hennen berücksichtigt wurden. Die mittleren Mortalitätsraten zeigt Tabelle 1. Die Abgangsrate in Gruppenkäfighaltung war mit 6,3\% etwa doppelt so hoch wie die mittlere Abgangsrate für die Einzelkäfighaltung.

Tabelle 1

Anzahl überlebende und abgegangene Hennen in Legemonat 1 bis 6 sowie zugehörige Frequenzen für die Mortalität innerhalb und über die Generationen (Number of hens survived and died in period 1 to 6 with corresponding frequencies of mortality between and over the generations).

\begin{tabular}{|c|c|c|c|c|c|c|}
\hline \multirow[b]{2}{*}{ Generation } & \multirow[b]{2}{*}{0} & \multicolumn{2}{|c|}{ Einzelkäfig(ek) } & \multicolumn{2}{|c|}{ Gruppenkäfig(gk) } & \multirow[b]{2}{*}{$* 100 \%$} \\
\hline & & 1 & $* 100 \%$ & 0 & 1 & \\
\hline 1 & 2312 & 50 & 2,12 & 2481 & 135 & 5,16 \\
\hline 2 & 3081 & 161 & 4,97 & 2273 & 199 & 8,05 \\
\hline 3 & 2961 & 71 & 2,34 & 1718 & 102 & 5,60 \\
\hline Summe & 8354 & 282 & 3,27 & 6472 & 436 & 6,31 \\
\hline
\end{tabular}

\section{3.} Methode

Die Mortalitätsdaten entsprechen einer binären Variablen $\mathrm{y}_{\mathrm{i}}$ mit den Ausprägungen $\mathrm{y}_{\mathrm{i}}=0$, falls Henne $\mathrm{i}$ die ersten sechs Legemonate überlebt hat und $\mathrm{y}_{\mathrm{i}}=1$, falls Henne $\mathrm{i}$ in diesen Monaten abgegangen ist. Die genetisch-statistische Analyse dieses $(0,1)$ Merkmals kann formal mit dem folgenden linearen Tiermodell erfolgen:

$$
y_{i}=x_{i}^{\prime} \beta+z_{i}^{\prime} a+e_{i} \quad \text { mit } \quad E\left(y_{i}\right)=x_{i}^{\prime} \beta \quad \operatorname{Var}\left(y_{i}\right)=z_{i}^{\prime} A z_{i} \cdot \sigma_{a}^{2}+\sigma_{e}^{2}
$$

Hierbei sind $\beta$ der Vektor der fixen Modelleffekte (hier von Generation, Haus, Schlupf und Reihe), a der Vektor der additiv-genetischen Effekte, A die zugehörige numerische Verwandtschaftsmatrix, $\sigma_{a}^{2}$ die genetische und $\sigma_{e}^{2}$ die umweltbedingte Varianz. Die Versuchplanvektoren $x_{i}^{\prime}$ und $z_{i}^{\prime}$ ordnen die entsprechenden Modelleffekte der Beobachtung $y_{i}$ zu. Das obige lineare Modell hat den Nachteil, dass Vorhersagen $\hat{y}_{i}$ nicht ausschließlich im Intervall $(0,1)$ liegen müssen. In der Tierzüchtung hat sich deshalb zur Auswertung von binären Merkmalen das Schwellenwertmodell etabliert. Bei diesem Modell wird unterstellt, dass der beobachtbaren binären Variable $\mathrm{y}_{\mathrm{i}}$ eine latente (nichtbeobachtbare) Zufallsvariable $y_{i}^{*}$ zugrunde liegt. Überschreitet die latente, stetige Variable $y_{i}^{*}$ einen Schwellenwert (gewöhnlich den Wert Null) so nimmt $\mathrm{y}_{\mathrm{i}}$ den Wert Eins ansonsten den Wert Null an. Es besteht also der Zusammenhang (vgl. FALCONER, 1984):

$$
y_{i}=\left\{\begin{array}{lll}
1 & \text { falls } & y_{i}^{*}>0 \\
0 & \text { falls } & y_{i}^{*} \leq 0
\end{array}\right\} \quad \text { mit } \quad y_{i}^{*}=x_{i}^{\prime} \beta+z_{i}^{\prime} a+e_{i}
$$

Setzt man voraus, dass die Resteffekte der latenten Zufallsgröße einer symmetrischen Verteilung mit der Verteilungsfunktion $\mathrm{F}(\mathrm{z})$ genügen, so folgt für den bedingten Erwartungswert die Darstellung:

$$
E\left(y_{i} \mid a\right)=0 \cdot P\left(y_{i}^{*} \leq 0\right)+1 \cdot P\left(y_{i}^{*}>1\right)=P\left(y_{i}^{*}>0\right)=F\left(\frac{x_{i}^{\prime} \beta+z_{i}^{\prime} a}{\sigma_{e}}\right)
$$

Aus obiger Darstellung folgt, dass nur der Quotient $\beta / \sigma_{\mathrm{e}}$ eindeutig identifizierbar ist. Folglich wird gewöhnlich $\sigma_{e}^{2}=1$ gesetzt (vgl. McCULLOCH and SEARLE, 2001). 
Die Verteilungsfunktion $\mathrm{F}(\mathrm{z})$, die sichert, dass für beliebige Werte von $\beta$ und $a$ der bedingte Erwartungswert von $\mathrm{y}_{\mathrm{i}}$ zwischen Null und Eins liegt, wird auch als inverse Linkfunktion bezeichnet. Am gebräuchlichsten sind die Verteilungsfunktion der standardisierten Normalverteilung und die logistische Funktion. Die zugehörigen Linkfunktionen werden als Probit- und Logitfunktionen bezeichnet. Im Fall der logistischen Funktion gilt:

$$
F(z)=\frac{\exp (z)}{1+\exp (z)} \quad \text { mit } \quad E(Z)=0 \text { und } \operatorname{Var}(Z)=\frac{\pi^{2}}{3}
$$

Der binären Variablen liegt also im Fall der Logitfunktion eine stetige Variable mit Restvarianz $\pi^{2} / 3=3,29$ und im Fall der Probitfunktion eine stetige Variable mit Restvarianz Eins zugrunde. Die Heritabilitäten müssen aus folgenden Beziehungen geschätzt werden.

$$
h_{L O G}^{2}=\frac{\sigma_{a}^{2}}{\sigma_{a}^{2}+\left(\pi^{2} / 3\right)} \quad \text { und } \quad h_{P R O}^{2}=\frac{\sigma_{a}^{2}}{1+\sigma_{a}^{2}}
$$

Zwischen dem geschätzten Zuchtwert von Tier i und der zugehörigen Abgangsrate $\mathrm{p}_{\mathrm{i}}$ besteht im linearen Modell ein direkter Zusammenhang während im Thresholdmodell eine Rücktransformation mit Hilfe der inversen Linkfunktion erfolgen muss. Es gilt:

$$
\hat{p}_{L A M, i}=x_{i}^{\prime} \hat{\beta}_{L A M}+\hat{a}_{L A M, i} \quad \text { und } \quad \hat{p}_{T A M, i}=F\left(x_{i}^{\prime} \hat{\beta}_{T A M}+\hat{a}_{T A M, i}\right)
$$

Ein Schwellenwertmodell der Gestalt: $y_{i j}^{*}=x_{i j}^{\prime} \beta+s_{i}+e_{i j}$, wobei $s_{i}$ der zufällige Effekt von Vater $i$ ist, wird nachfolgend im Gegensatz zum „threshold animal model“ (TAM) als „threshold sire model“ (TSM) bezeichnet. In Erweiterung der Abkürzungen für die Einmerkmalsmodelle bezeichnet LLAM ein bivariates lineares Tiermodell während die Abkürzung TTAM (bzw. TTSM) für ein bivariates Schwellenwerttier- (bzw. Schellenwertvatermodell) steht.

Zur Schätzung der fixen Modelleffekte, der Vorhersage der Zuchtwerte und zur Schätzung der Varianzkomponenten wurde das ASREML-Programm von GILMOUR u.a. (2002) verwendet, wobei aus Konvergenzgründen die Logitfunktion zum Einsatz kam. Da in ASREML standardmäßig nur die gemeinsame Analyse eines binären und eines stetigen Merkmals möglich ist, wurde die genetische Analyse der ThresholdThreshold-Modelle über univariate Analysen mit spezieller Struktur der genetischen und der umweltbedingten Varianz-Kovarianz-Matrix realisiert. Durch Einführung von Missing values wurden dazu die Datensätze aus den Einzel- und Gruppenkäfigen zu Paaren von insgesamt 15544 Beobachtungen ergänzt. Zusätzlich wurde eine neue Spalte (row) mit 1 und 2 als Indikator eingeführt, um in jedem Wertepaar das entsprechende Merkmal identifizieren zu können. Für die Resteffekte der latenten Variable gilt dann: e $\sim \mathrm{N}\left(0, I_{15544} \otimes R_{2}\right)$ wobei $R_{2}$ die umweltbedingte Varianz-Kovarianz-Matrix (VCM) für die Wertepaare und $\otimes$ das Kroneckerprodukt sind. Das Modell enthält jeweils 2 additiv-genetische Effekte für insgesamt 16921 Pedigreetiere. Bezeichne A die zugehörige numerische Verwandtschaftsmatrix. Dann ergibt sich:

row.animal $\sim N\left(0, G_{2} \otimes I_{16921} \cdot A\right)$ mit $G_{2}$ genetische VCM der beiden Abgangsraten. Bei der Maximierung der Likelihood mit ASREML unter Annahme einer binären Variablen sind die beiden Restvarianzen mit Eins fest vorzugeben. Da weiterhin nicht simultan 2 Beobachtungen am gleichen Tier vorliegen können, muss zusätzlich die Kovarianz der Resteffekte mit Null fixiert werden. 
4. Ergebnisse und Diskussion

4.1. Heritabilitäten und Abgangswahrscheinlichkeiten

Die genetischen Parameter geschätzt mit einem linearen Zweimerkmalsmodell und bivariaten Schwellenwertmodellen enthält Tabelle 2.

Mit dem linearen Tiermodell (LLAM) wurden für die Mortalität im Einzel- bzw. Gruppenkäfig eine Heritabilität von 0,015 bzw. von 0,032 und eine genetische Korrelation von 0,574 geschätzt.

Tabelle 2

Heritabilitäten und genetische Korrelationen für die Mortalität in Einzel- und Gruppenkäfigen geschätzt mit linearen und Schwellenwert-Modellen (Heritabilities and genetic correlations of mortality in single and group cages estimated with linear and threshold models)

\begin{tabular}{lcccc}
\hline \multicolumn{1}{c}{ Modelle } & \multicolumn{3}{c}{$\mathrm{h}^{2} \pm \mathrm{se}$} \\
& Abkürzung & Einzelkäfig & Gruppenkäfig & $\mathrm{r}_{\mathrm{g}} \pm \mathrm{se}$ \\
\hline linear-linear animal & (LLAM) & $0,015 \pm 0,008$ & $0,032 \pm 0,012$ & $0,574 \pm 0,305$ \\
threshold-threshold animal & (TTAM) & $0,096 \pm 0,048$ & $0,099 \pm 0,037$ & $0,680 \pm 0,377$ \\
threshold-threshold sire & (TTSM) & $0,134 \pm 0,091$ & $0,212 \pm 0,078$ & $0,697 \pm 0,369$ \\
\hline
\end{tabular}

Das Verhältnis der Heritabilitäten im linearen Modell widerspiegelt die Relation der mittleren Abgangsraten, die im Einzelkäfig bei 3,3\% und in Gruppenkäfig bei 6,3\% lagen. Mit den Schwellenwertmodellen wurden im Vergleich zu den linearen Modellen generell höhere Heritabilitäten und leicht erhöhte genetische Korrelationen geschätzt. Während mit dem Threshold Sire-Modell für die Mortalität im Einzel- und Gruppenkäfig unterschiedliche Werte gefunden wurden, lieferte das Threshold Animal-Modell für beide Merkmale mit 0,096 bzw. 0,099 sehr ähnliche Schätzwerte.

Unter Verwendung der Formeln für die Vorhersage von Abgangswahrscheinlichkeiten aus Abschnitt 3 wurden die Abgangsraten für alle Hennen und für die 10\%-besten Hennen (bei Einschluss der abgegangenen Tiere) in der Population ermittelt. In Tabelle 3 ist die zugehörige beschreibende Statistik aufgeführt. Die in Tabelle 3 zusammengestellten Ergebnisse zeigen die Nachteile des linearen Modellansatzes im Vergleich zum Schwellenwertmodell bei Verwendung von entsprechenden Linkfunktionen auf. Im Mittel über alle Tiere werden innerhalb der Modellklassen gleiche Abgangsfrequenzen vorhergesagt. Während jedoch im Schwellenwertmodell die Minima generell positiv sind weisen die Abgangsraten vorhergesagt mit dem linearen Modell für bestimmte Tiere negative Werte auf.

Tabelle 3

Geschätzte Wahrscheinlichkeiten für den Abgang aller Hennen und der 10\%-besten Hennen (bei Annahme des Threshold-Modells) im Einzel-(ab_ek) und Gruppenkäfig (ab_gk) (Estimated probabilities of death for all and the $10 \%$ best hens in single and group cages)

\begin{tabular}{lcccccc}
\hline & \multicolumn{3}{c}{ ab_e (N=8636) } & \multicolumn{3}{c}{ ab_e (N=864) } \\
Modell & Mean & Min & Max & Mean & Min & Max \\
\hline LAM & 0.0327 & -0.0021 & 0.1023 & 0.0268 & -0.0021 & 0.0650 \\
TAM & 0.0327 & 0.0092 & 0.1332 & 0.0284 & 0.0092 & 0.0598 \\
\hline & & ab_k (N=6908) & & & ab_k (N=691) & Max \\
Modell & Mean & Min & Max & Mean & Min & 0.0711 \\
LAM & 0.0631 & 0.0164 & 0.1493 & 0.0407 & 0.0164 & 0.0711 \\
TAM & 0.0631 & 0.0296 & 0.1593 & 0.0468 & 0.0296 & \\
\hline
\end{tabular}

\subsection{Rangkorrelationen zwischen Vater- und Mutterzuchtwerten}

Das vordergründige Anliegen einer Zuchtwertschätzung ist primär die Rangierung der Tiere anhand der geschätzten Zuchtwerte zur Auswahl der Elterntiere und nur sekun- 
där die Vorhersage von tier- oder gruppenspezifischen Abgangswahrscheinlichkeiten. Deshalb werden nachfolgend ausgewählte Rangkorrelationen zwischen den Zuchtwerten von Vätern geschätzt mit den drei untersuchten Ein- und Zweimerkmalsmodellen dargestellt.

Da die verwendete inverse Linkfunktion als Verteilungsfunktion zwangsläufig streng monoton wachsend ist, kann die Rangordnung der Tiere nach den geschätzten Zuchtwerten für die latente Variable erfolgen. Je kleiner der geschätzte Zuchtwert desto geringer fällt die zugehörige durch Rücktransformation erhältliche Abgangsfrequenz aus.

\section{Tabelle 4}

Rangkorrelationen zwischen den Zuchtwerten aller Väter (bzw. Mütter), den 10\%-und den 5\%-besten Vätern (bzw. Müttern) für die Abgangsrate im Einzel- und Gruppenkäfig (Rank correlations between breeding values of all, the $10 \%$ best and the $5 \%$ best sires and dams for death rate in single and group cages)

\begin{tabular}{|c|c|c|c|c|c|c|c|}
\hline \multirow{3}{*}{\multicolumn{2}{|c|}{ Anzahl der Väter: }} & \multicolumn{6}{|c|}{ threshold animal model (TAM) } \\
\hline & & \multicolumn{3}{|c|}{ ab_ek } & \multicolumn{3}{|c|}{ ab_gk } \\
\hline & & 236 & 24 & 12 & 229 & 23 & 11 \\
\hline \multirow{2}{*}{$\begin{array}{l}\text { linear animal model } \\
\text { threshold sire model }\end{array}$} & $(\mathrm{LAM})$ & 0,998 & 0,972 & 0,958 & 0,996 & 0,962 & 0,982 \\
\hline & (TSM) & 0,810 & 0,665 & 0,783 & 0,921 & 0,489 & 0,564 \\
\hline \multicolumn{2}{|c|}{ Anzahl der Mütter: } & 1758 & 176 & 88 & 1707 & 171 & 85 \\
\hline linear animal model & (LAM) & 0,999 & 0,980 & 0,943 & 0,996 & 0,913 & 0,933 \\
\hline
\end{tabular}

Die Rangkorrelationen zwischen den Vaterzuchtwerten geschätzt mit den drei Einmerkmalsmodellen separat für die Mortalität im Einzel- und Gruppenkäfig sind in Tabelle 4 aufgelistet. Die Zuchtwerte geschätzt mit dem linearen und dem Threshold Animal-Modell zeigen für die Mortalität im Einzel- und im Gruppenkäfig mit Werten zwischen 0,958 und 0,998 sowohl für alle Väter als auch für die Väter mit den geringsten Mortalitätszuchtwerten sehr hohe Rangkorrelationen. Dagegen liegen die Rangkorrelationen zwischen dem Referenzmodell und dem Threshold Sire-Modell für die 10\%-besten Väter mit 0,665 und 0,489 nur auf mittlerem Niveau. Mit Werten von 0,943 und 0,933 fallen die Rangkorrelationen zwischen den 5\%-besten Müttern für die Modelle LAM und TAM etwas geringer aus. Die Ursache liegt wahrscheinlich in der geringeren Anzahl Töchter je Mutter im Vergleich zu der höheren Anzahl Töchter je Vater und ist somit in der Genauigkeit der Zuchtwertschätzung zu suchen.

Um die Güte der untersuchten Modelle, insbesondere aber die der linearen Modelle im Vergleich zu den Schwellenwertmodellen einzuschätzen, wurde das bivariate Schwellenwerttiermodell als Referenzmodell angesehen. Tabelle 5 enthält die geschätzten Rangkorrelationen aller untersuchten Ein- und Zweimerkmalsmodelle bezogen auf diese Referenz.

Tabelle 5

Rangkorrelationen zwischen den Zuchtwerten aller Väter und den 10\%-besten Vätern separat für die Abgangsrate im Einzel- und Gruppenkäfig bezogen auf das Zweimerkmals-Schwellenwerttiermodell (Rank correlations between the breeding values of all and the $10 \%$ best sires for death rate in single and group cages refer to the two-trait threshold model).

\begin{tabular}{|c|c|c|c|c|c|}
\hline \multirow[b]{3}{*}{ Modelle } & \multicolumn{5}{|c|}{ threshold-threshold animal model (TTAM) } \\
\hline & \multirow[t]{2}{*}{ Abkürzung } & \multicolumn{2}{|c|}{ ab_ek } & \multicolumn{2}{|c|}{ ab_gk } \\
\hline & & $\mathrm{N}=229$ & $\mathrm{~N}=23$ & $\mathrm{~N}=229$ & $\mathrm{~N}=23$ \\
\hline linear animal & (LAM) & 0,846 & 0,227 & 0,912 & 0,823 \\
\hline threshold animal & (TAM) & 0,850 & 0,191 & 0,917 & 0,809 \\
\hline threshold sire & (TSM) & 0,757 & 0,209 & 0,814 & 0,661 \\
\hline linear-linear animal & (LLAM) & 0,992 & 0,832 & 0,992 & 0,986 \\
\hline threshold-threshold sire & (TTSM) & 0,846 & 0,488 & 0,889 & 0,729 \\
\hline
\end{tabular}


Innerhalb der Einmerkmalsmodelle lieferten das lineare und das Threshold-Animalmodell fast identische Rangkorrelationen zum Referenzmodell. Die Rangkorrelationen des linearen Zweimerkmalsmodells zeigten für alle Väter in beiden Abgangsraten mit 0,992 sehr hohe Werte. Auch für die 10\%-besten Väter lieferte dieses Modell mit 0,832 und 0,986 im Vergleich zu den Alternativen die höchsten Werte. Diese Konstellation belegt, analog zu den Ergebnissen aus Tabelle 4, die gute Überstimmung des linearen und des Schwellenwertmodells falls lediglich eine Rangierung der Tiere nach ihren Zuchtwerten durchgeführt werden soll. Die Schwächen des linearen Modells bei der Vorhersage von tierspezifischen Abgangswahrscheinlichkeiten wirken sich nur sehr geringfügig auf die richtige Rangordnung der Tiere aus.

Bisher wurde der Modellvergleich nur innerhalb der Abgangsraten von Einzel- und Gruppenkäfig durchgeführt. In Tabelle 6 sind für alle untersuchten Modelle Rangkorrelationen zwischen den Mortalitätszuchtwerten im Einzel- und Gruppenkäfig dargestellt.

Tabelle 6

Rangkorrelationen der Zuchtwerte aller Väter (mit Nachkommen sowohl im Einzel- als auch im Gruppenkäfig) zwischen den Abgangsraten für Einzel- und Gruppenkäfig (Rank correlations of breeding values for all sires between death rates in single and group cages)

\begin{tabular}{lllcccc}
\hline & & \multicolumn{6}{c}{ ab_gk (N=229) } \\
ab_ek & LAM & TAM & TSM & LLAM & TTAM & TTSM \\
\hline TAM & 0,191 & 0,207 & 0,098 & 0,458 & 0,539 & 0,320 \\
TTAM & 0,641 & 0,649 & 0,535 & 0,838 & 0,886 & 0,710 \\
\hline
\end{tabular}

Die Modelle LAM und TAM (bzw. LLAM und TTAM) liefern wiederum sehr ähnliche Korrelationen zwischen den Zuchtwerten für die Mortalität im Einzel- und im Gruppenkäfig. Im Vergleich zum TSM und TTSM zeigen die beiden linearen Tiermodelle wiederum höhere korrelative Beziehungen zu den beiden Schwellenwert-Tiermodellen. Die Rangkorrelationen zwischen den Zuchtwerten für die Mortalität im Einzel- und im Gruppenkäfig bei Schätzung jeweils mit Einmerkmalmodellen sind mit 0,191, 0,207 bzw. 0,098 für LAM, TAM und TSM sehr niedrig. Diese geringen Werte sind Ausdruck der niedrigen Heritabilitäten, der moderaten genetischen Korrelation der Abgangsraten und der Anzahl Nachkommen pro Vater im Einzel- und im Gruppenkäfig. Durch simultane Zuchtwertschätzung mit dem Modell TTAM lässt sich eine Erhöhung der Rangkorrelation auf 0,886 erreichen. Die Ergebnisse aus Tabelle 6 verdeutlichen die Notwendigkeit einer gemeinsamen Zuchtwertschätzung für die Mortalität in Einzel- und Gruppenkäfighaltung. Für eine solche Zuchtwertschätzung stellt das bivariate lineare Modell einen brauchbaren Kompromiss dar, zumal sich dieses Modell problemlos um weitere Merkmale erweitern lässt. Aus theoretischen Gründen sollte ein multivariates Modell mit mehreren Schwellenwert- und stetigen Merkmalen das Modell der Wahl sein. Man muss aber bedenken, dass in derartigen Modellen sehr viele Parameter zu schätzen sind. Auch wenn die entsprechenden Algorithmen und Programme vorhanden sind, können Konvergenzprobleme, die sich bei dieser Studie bereits für die univariaten Thresholdmodelle zeigten, die Rückkehr zu einfacheren lediglich suboptimalen Modellen bewirken. 


\section{Danksagung}

Dr. Luc Janss aus der Gruppe für Tierzucht und Genetik der Universität Wageningen sei herzlich für die Unterstützung bei der Auswertung der Daten mit Schwellenwertmodellen unter Verwendung von ASREML gedankt.

\section{Literatur}

DUCROCQ, V.; BESBES, B.; PROTAIS, M.:

Genetic improvement of laying hens viability using survival analysis. Genet. Sel. Evol. 32 (2000), 2340

FALCONER, D.S.:

Einführung in die Quantitative Genetik. (Aus dem Englischen von P. Glodek), Verlag Eugen Ulmer Stuttgart, 1984

GILMOUR, A.R.; CULLIS, B.R.; WELHAM, S.J.; THOMPSON, R.: ASReml Reference Manual, 2nd edition (2002)

MCCULLOCH, C.E.; SEARLE, S.R.:

Generalized, Linear and Mixed Models. John Wiley \& Sons, Inc. (2001)

MIELENZ, N.; NURGIARTININGSIH, V.M.A.; SCHMUTZ, M.; SCHÜLER, L.: Schätzung von Varianzkomponenten mit Gruppenmittelwerten am Beispiel von Legehennen, gehalten in Gruppenkäfigen. Arch. Tierz., Dummerstorf 46 (2003) 5, 491-498

NURGIARTININGSIH, V.M.A.; MIELENZ, N.; PREISINGER, R.; SCHMUTZ, M.; SCHÜLER, L.: Genetic parameters for egg production and egg weight of laying hens housed in single and group cages. Arch. Tierz., Dummerstorf 45 (2002) 5, 501-508

NURGIARTININGSIH, V.M.A.; MIELENZ, N.; PREISINGER, R.; SCHMUTZ, M.; SCHÜLER, L.: Estimation of genetic parameters based on individual and group mean records in laying hens. British Poultry Science, 45 (2004) 5, 604-610

PREISINGER, R.:

Internationalisation of breeding programmes - breeding egg-type chickens for a global market. $6^{\text {th }}$ World Congress on Genetics Applied to Livestock Production, 26 (1998), 135-142 Armindale, Australia

ROBERTSON, A.; LERNER, M.:

The heritability of all-or-none trait: viability of poultry. Genetics 34 (1949), 395-411

SAVAS, T.; PREISINGER, R.; RÖHE, R.; KALM, E.:

Genetische Parameter und optimale Prüfdauer für Legeleistung anhand von Teillegeleistungen bei Legehennen. Arch. Tierz., Dummerstorf 41 (1998) 5, 421-432

Eingegangen: 02.05.2005

Akzeptiert: 15.06.2005

Autor für Korrespondenz

Dr. NORBERT MIELENZ

Institut für Tierzucht und Tierhaltung mit Tierklinik der

Martin-Luther-Universität Halle-Wittenberg

Adam-Kuckhoff-Straße 35

06108 HALLE

E-Mail: norbert.mielenz@landw.uni-halle.de 